\title{
L'horloge biologique du muscle
}

\author{
Alicia Mayeuf-Louchart
}

> L'horloge biologique joue un rôle essentiel dans le contrôle de l'activité musculaire, en dissociant temporellement les fonctions métaboliques du muscle squelettique. Les capacités musculaires en réponse à l'exercice sont également circadiennes. Des perturbations des rythmes biologiques, telles que celles retrouvées chez les travailleurs postés affectent la fonction musculaire et sont associées au développement de la sarcopénie. <

L'horloge biologique est un système ancestral permettant aux organismes d'anticiper les variations journalières de l'environnement. Chaque organe possède sa propre horloge biologique et l'ensemble de ces horloges est synchronisé par l'horloge centrale, localisée dans les noyaux suprachiasmatiques de l'hypothalamus, elle-même sous l'influence de la lumière. Les rythmes circadiens (du latin : circa pour autour et dies pour jour) sont générés grâce à un circuit moléculaire, appelé horloge moléculaire, qui se compose de facteurs de transcription régulant l'expression de leurs gènes cibles, dont leurs propres régulateurs (Figure $1 \mathrm{~A}$ ). Des boucles transcriptionnelles et traductionnelles de rétrocontrôles gouvernent ainsi l'expression circadienne de nombreux gènes dans toutes les cellules. La plupart des fonctions physiologiques sont soumises à des régulations circadiennes. Par conséquent, la perturbation des rythmes circadiens est à l'origine de nombreuses pathologies, telles que certaines maladies neurodégénératives, des cancers et certains désordres métaboliques [1]. Le muscle squelettique représente plus de $40 \%$ de la masse corporelle et est responsable de la capture de $80 \%$ du glucose postprandial. Il joue donc un rôle majeur dans la régulation du métabolisme général.

\section{Contrôle circadien du métabolisme musculaire}

L'horloge biologique du muscle squelettique contrôle l'expression de gènes codant des protéines majoritairement impliquées dans les processus métaboliques. Des études transcriptomiques réalisées chez la souris ont montré que 1359 gènes «cyclent» dans le Soleus (muscle lent) alors que seulement 684 gènes oscillent dans le jambier antérieur (muscle mixte). Ceci souligne l'importance de l'horloge biologique dans la régulation du métabolisme musculaire [2]. L'horloge biologique permet la séparation temporelle de l'utilisation des substrats énergétiques au cours de la journée (pour revue [3]). Dans le muscle, l'utilisation des glucides a ainsi lieu au début de la phase active, alors que les lipides sont stockés en fin de période active et sont oxydés au cours de la période inactive, où ils demeurent le principal substrat énergétique. La mitochondrie joue un rôle essentiel dans le lien entre horloge biologique et métabolisme, et la plupart des études menées sur les mutants des gènes de l'horloge chez la souris ont démontré des perturbations de la fonction mitochondriale. Notre équipe a, par exemple, montré que la délétion du gène de l'horloge Rev-erb $\alpha$ chez la souris affectait la biogenèse et la respiration mitochondriale dans le muscle (Figure 1B) [4]. Chez l'Homme, la respiration mitochondriale présente également des fluctuations diurnes qui atteignent leur maximum vers 23 heures [5].

\section{Horloge musculaire et exercice}

L'activité musculaire est contrôlée par l'horloge biologique. Elle se restreint à la phase dite «active», durant laquelle l'organisme est éveillé. De nombreuses études ont tenté de déterminer si les performances musculaires varient en fonction de l'heure de la journée à laquelle est réalisé un exercice. L'ensemble de ces études s'accordent pour montrer que celle-ci est optimale lorsque l'exercice est réalisé entre 16 et 18 heures chez l'Homme (Figure 1C) (pour revue [6]). II est toutefois important de prendre en considération le chronotype de l'individu. Ce paramètre se mesure en fonction de l'heure du coucher, le temps de sommeil et l'heure de réveil, et permet un classement en trois groupes (tôt, intermédiaire, tard). Il existe par ailleurs un mécanisme d'anticipation de l'horloge biologique, qui prépare le muscle à 
A

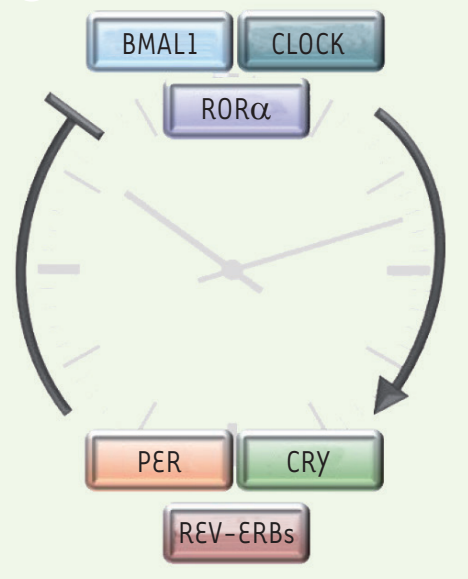

B

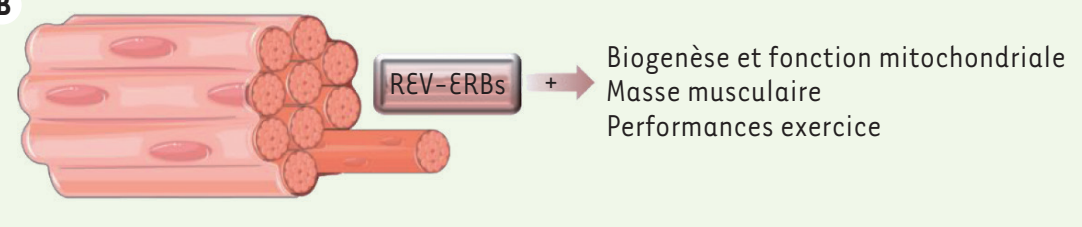

C

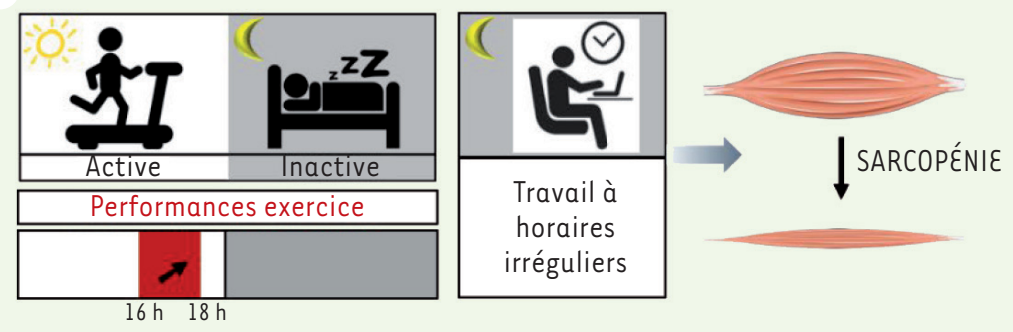

Figure 1. Horloge biologique dans le muscle squelettique. A. Le circuit moléculaire de l'horloge, qui s'exprime dans toutes les cellules, est composé

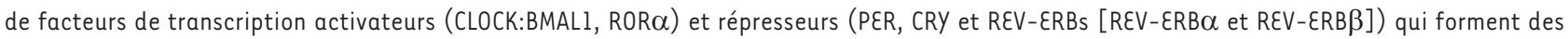
boucles de rétroaction transcriptionnelle. Cette horloge génère des fluctuations autonomes de la transcription et de la traduction sur une période de 24 heures. B. Fonctions de REV- $\varepsilon R B \alpha$ dans le muscle squelettique décrites par notre équipe. C. L'activité musculaire est circadienne et des perturbations de l'horloge biologique sont liées au développement de la sarcopénie.

l'exercice lorsque l'entrainement a lieu à une heure régulière (pour revue [6]). Les signatures transcriptomiques et métaboliques du muscle sont différentes en fonction de l'heure à laquelle l'exercice est réalisé [7]. En effet, un exercice réalisé au début de la phase inactive stimule l'oscillation des gènes et des métabolites liés au métabolisme des glucides et atténue celle liée au métabolisme du glycérol, alors qu'un exercice réalisé en début de phase active abolit l'oscillation rythmique des gènes et métabolites liés au métabolisme des glucides. Par conséquent, l'heure à laquelle est effectué un exercice retentit sur le métabolisme du muscle squelettique, et donc sur le métabolisme général, ce qui peut être à l'origine de désordres métaboliques.

Réciproquement, le laboratoire du $\operatorname{Pr} K$. Esser a récemment montré que l'exercice agit sur l'horloge biologique du muscle et peut être ainsi considéré comme un signal synchronisateur (appelé Zeitgeber) [8]. Dans cette étude, les auteurs ont soumis des souris à une session d'exercice à trois moments de la journée. Les résultats montrent qu'in vivo, une seule séance d'exercice suffit à modifier la phase du rythme circadien musculaire. En réalisant des stimulations électriques sur des myotubes in vitro (C2C12) à différents moments après leur synchronisation en culture, les auteurs montrent également que la contraction musculaire est suffisante pour médier cet effet, indépendamment des facteurs systémiques. Notre équipe a également montré que chez la souris, l'exercice induit l'augmentation de l'expression du gène de I'horloge Rev-erb $\alpha$ et que l'activation pharmacologique de Rev-erb $\alpha$ améliore les capacités à l'exercice (Figure 1B) [4]. Chez l'Homme, une seule séance d'exercice est également suffisante pour moduler l'expression des gènes de l'horloge biologique (pour revue [9]).

L'ensemble de ces travaux indique donc qu'il existe un lien à double sens entre horloge musculaire et exercice. L'activité et les performances sont sous le contrôle de l'horloge et en retour l'exercice module l'horloge musculaire.

\section{Glucocorticoïdes et horloge musculaire}

Certains facteurs sont connus comme étant capables de moduler l'horloge biologique tels que les glucocorticoïdes. Les niveaux circulants de glucocorticoïdes présentent en effet une rythmicité circadienne, avec des niveaux maximaux en début de phase active. Réciproquement, la dexaméthasone - un glucocorticoïde synthétique - est un puissant synchronisateur dans les tissus périphériques puisque des éléments de réponse au récepteur des glucocorticoïdes sont présents dans les promoteurs des gènes de l'horloge. L'administration chronique de dexaméthasone induit une atrophie musculaire, tout comme la délétion du gène de l'horloge $\operatorname{Rev-erb} \alpha$. Dans cette étude, nous avons montré que l'induction génétique ou l'activation pharmacologique de REV-ERB $\alpha$ corrige l'atrophie musculaire induite par les glucocorticoides [10]. Ces données soulignent donc l'importance de la chronopharmacologie dans le traitement des maladies musculaires.

\section{Horloge biologique et sarcopénie}

Alors que 15 à $30 \%$ de la population mondiale travaille en horaires décalés, des études montrent que le risque de développer une sarcopénie est supérieure dans ces 
populations (Figure 1C) (pour revue [9]). La sarcopénie est une perte de la masse et de la force musculaires qui intervient au cours du vieillissement physiologique. De nombreux travaux ont mis en avant le lien existant entre horloge biologique et vieillissement dans différents modèles animaux. C'est le cas chez la drosophile où une désynchronisation chronique de l'horloge raccourcit la durée de vie. Des études réalisées chez des souris génétiquement déficientes pour des gènes de l'horloge ont également abouti à une réduction de la durée de vie associée à l'apparition d'une sarcopénie précoce. La mitochondrie joue un rôle important dans le développement de la sarcopénie induite par une dérégulation de l'horloge biologique (pour revue [9]). Cependant, la délétion du gène de l'horloge Bmall spécifiquement dans le muscle squelettique n'induit pas ce vieillissement prématuré du muscle, suggérant donc que des facteurs indépendants de la fibre musculaire sont aussi à l'origine de ce phénomène (pour revue [11]).

L'exercice musculaire est reconnu comme étant une stratégie préventive et thérapeutique de la sarcopénie, capable d'atténuer la perte de masse et de force musculaires. La prescription d'exercices personnalisés à certaines heures pourrait permettre d'optimiser les effets bénéfiques de l'exercice pour lutter contre la progression de la sarcopénie liée à l'âge et/ou à des dérégulations de l'horloge biologique. Ces mesures pourraient également être appliquées à titre préventif pour limiter l'apparition de la sarcopénie chez les travailleurs soumis à horaires irréguliers.

\section{Horloge biologique et régénération musculaire}

Le vieillissement du muscle est également associé à une diminution de sa capacité à régénérer. Dans le muscle, les cellules souches musculaires, également nommées cellules satellites, permettent la régénération des fibres endommagées. Cependant, leur capacité à régénérer diminue au cours du vieillissement [12]. En 2017, le laboratoire du Pr Muñoz-Cánoves a réalisé une étude transcriptomique sur des cellules souches musculaires, isolées toutes les 4 heures pendant 24 heures, à partir de souris jeunes et âgées ( $\geq 18$ mois) [13]. Ces études montrent qu'alors que l'amplitude et la période du rythme circadien de l'expression des gènes de l'horloge ne changent pas au cours du vieillissement, le transcriptome rythmique de ces cellules est, lui, reprogrammé. Des gènes impliqués dans les fonctions homéostatiques perdent ainsi leur rythmicité, alors que des gènes qui ne "rythmaient" pas se mettent à "cycler" dans des cellules âgées. C'est par exemple le cas de familles de gènes impliquées dans la réparation de I'ADN mitochondrial ou dans l'inflammation. Les auteurs montrent que la restriction calorique est capable de prévenir cette reprogrammation métabolique des cellules souches musculaires âgées. Toutefois, à l'heure actuelle, les mécanismes par lesquels l'horloge biologique régulerait la fonction des cellules souches musculaires ne sont pas connus.

L'horloge biologique contrôle aussi la régénération de l'intestin et de la peau, où il a été montré qu'une blessure induite pendant la phase active régénère $60 \%$ plus vite que lorsqu'elle est induite durant la phase inactive [14]. La régénération musculaire repose sur l'interaction de nombreux types cellulaires telles que les cellules souches musculaires, les cellules inflammatoires, les fibroblastes, ainsi que les fibres musculaires saines et endommagées. Mieux comprendre le rôle de l'horloge

biologique dans ces différents compartiments cellulaires contribuera donc au développement de chronothérapies appliquées dans le cadre de blessures, de pathologies ou du vieillissement musculaires. $\diamond$

\section{SUMMARY}

The muscle biological clock

The biological clock plays an essential role in the control of muscle activity, by dissociating temporally the metabolic functions of skeletal muscle. Exercise capacity also displays a circadian rhythm. Alterations in biological rhythm, as in shift workers, alter muscle function and are associated with the development of sarcopenia. $\diamond$

\section{LIENS D'INTÉRÊT}

L'auteure déclare n'avoir aucun lien d'intérêt concernant les données publiées dans cet article.

\section{NDLR}

L'auteure, Alicia Mayeuf-Louchard, a reçu le prix Impulsion de la Société Française de Myologie en 2019 pour l'ensemble de ses travaux de recherche.

\section{RÉFÉRENCES}

1. Mayeuf-Louchart A, Zecchin M, Staels B, et al. Circadian control of metabolism and pathological consequences of clock perturbations. Biochimie 2017 ; 143 : 42-50.

2. Dyar KA, Ciciliot $S$, Wright $L \varepsilon$, et al. Muscle insulin sensitivity and glucose metabolism are controlled by the intrinsic muscle clock. Mol. Metab 2014; $3: 29-41$.

3. Gutierrez-Monreal MA, Harmsen JF, Schrauwen P, et al. Ticking for metabolic health: the skeletal-muscle clocks. Obes. Silver Spring Md 2020;28 (suppl 1) : S46-54.

4. Woldt $\varepsilon$, Sebti Y, Solt LA, et al. Rev-erb- $\alpha$ modulates skeletal muscle oxidative capacity by regulating mitochondrial biogenesis and autophagy. Nat Med $2013 ; 19$ : 1039-46.

5. Moorsel D van, Hansen J, Havekes B, et al. Demonstration of a day-night rhythm in human skeletal muscle oxidative capacity. Mol Metab 2016 ; 5 : 635-45.

6. Mayeuf-Louchart A, Staels B, Duez H. Skeletal muscle functions around the clock. Diabetes Obes Metab $2015 ; 17$ (suppl 1) : 39-46.

7. Sato S, Basse AL, Schönke M, et al. Time of exercise specifies the impact on muscle metabolic pathways and systemic energy homeostasis. Cell Metab $2019 ; 30$ : $92-110 . e 4$.

8. Kemler D, Wolff CA, Esser KA. Time-of-day dependent effects of contractile activity on the phase of the skeletal muscle clock. J Physiol 2020 ; 598 : 3631-44.

9. Choi Y, Cho J, No MH, et al. Re-setting the circadian clock using exercise against sarcopenia. Int J Mol Sci $2020 ; 21: 3106$.

10. Mayeuf-Louchart A, Thorel $Q$, Delhaye $S$, et al. Rev-erb- $\alpha$ regulates atrophyrelated genes to control skeletal muscle mass. Sci Rep $2017 ; 7: 14383$.

11. Schiaffino S, Blaauw B, Dyar KA. The functional significance of the skeletal muscle clock: lessons from Bmall knockout models. Skelet Muscle $2016 ; 6: 33$.

12. Mayeuf $A$, Relaix F. Notch pathway: from development to regeneration of skeletal muscle. Med Sci (Paris) 2011 ; 27 : 521-6.

13. Solanas $G$, Peixoto FO, Perdiguero $\varepsilon$, et al. Aged stem cells reprogram their daily rhythmic functions to adapt to stress. Cell $2017 ; 170$ : 678-92.e20.

14. Hoyle NP, Seinkmane $\varepsilon$, Putker M, et al. Circadian actin dynamics drive rhythmic fibroblast mobilization during wound healing. Sci Transl Med 2017 ; 9 ; eaal2774.

TIRÉS À PART

A. Mayeuf-Louchard 